\title{
Energy-efficient Dual-codebook-based Backscatter Communications for Wireless Powered Networks
}

\author{
YUFAN ZHANG, School of Computer Science and Technology, Zhejiang University of Technology, China \\ ERTAO LI, School of Computer Science and Technology, Hangzhou Dianzi University, China \\ YI-HUA ZHU, School of Computer Science and Technology, Zhejiang University of Technology, China
}

\begin{abstract}
The common backscatter communications (ComBC), widely applied in wireless powered networks such as the RFID systems, exhibit the shortcoming that only a few bits are backscattered at a time due to energy limitation. It is significant to improve energy efficiency in backscatter communications so more data can be delivered within one backscatter. In this article, the energy-efficient dual-codebook based backscatter communications (DBBC) is proposed, which adopts two prefix codebooks shared by the sender and the receiver over a backscatter communication link. With the DBBC, the sender backscatters codewords from which the receiver decodes the original data. Using Energy Consumption Disparity (ECD) between backscattering bits 1 and 0 in the existing backscatter communications, we formulate the optimization problem minimizing energy consumption over the link for the DBBC. Mapping a prefix codebook into a binary tree and performing pruning and expanding operation (PEO) on binary trees, we obtain the solution to the optimization problem, which includes the two energy-efficient codebooks and the other two key parameters for the DBBC. The experiments on wireless identification sensing platform (WISP) show that, under the proposed DBBC, the sender can backscatter data $83 \%$ more than the ComBC with the same energy without sacrificing throughput.
\end{abstract}

CCS Concepts: • Networks $\rightarrow$ Network protocols; Network algorithms;

Additional Key Words and Phrases: Backscatter communications, wireless powered networks, energy conservation, prefix code

\section{ACM Reference format:}

Yufan Zhang, Ertao Li, and Yi-Hua Zhu. 2020. Energy-efficient Dual-codebook-based Backscatter Communications for Wireless Powered Networks. ACM Trans. Sen. Netw. 17, 1, Article 9 (November 2020), 20 pages. https://doi.org/10.1145/3426885

\section{INTRODUCTION}

In wireless powered networks, backscatter communications are frequently applied in delivering data. The common backscatter communications (ComBC) in the RFID systems [7] only backscatter a few bits at a time because of energy limitation. It is significant to design a backscatter communication scheme that can deliver more data within one backscatter.

This work was supported by the National Natural Science Foundation of China under Grants 61432015 and 61772470. Authors' addresses: Y. Zhang and Y.-H. Zhu (corresponding author), School of Computer Science and Technology, Zhejiang University of Technology, 286 Liuhe Road, Hangzhou, Zhejiang, 310023, China; emails: yufan-zhang@qq.com, yhzhu@zjut.edu.cn; E. Li, School of Computer Science and Technology, Hangzhou Dianzi University, No.1158, 2nd Road, Baiyang Street, Qiantang District, Hangzhou, Zhejiang, 310018, China; email: tonylee@hdu.edu.cn.

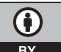

This work is licensed under a Creative Commons Attribution International 4. 0 License.

(c) 2020 Copyright held by the owner/author(s).

1550-4859/2020/11-ART9

https://doi.org/10.1145/3426885 


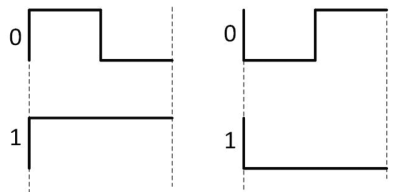

(a) FM0 symbols.
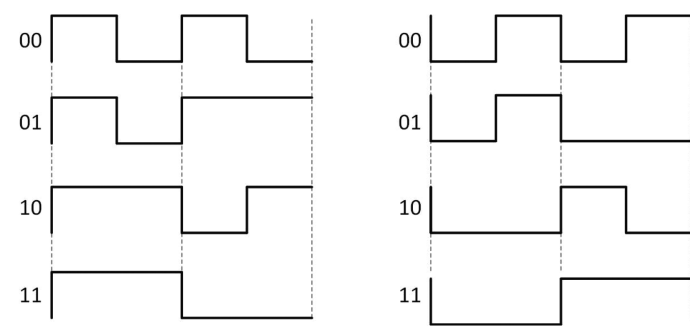

(b) FM0 sequences.

Fig. 1. FM0 (bi-phase) baseband encoding [7].

Backscatter communications deliver a bit after another based on some carrier-modulation or baseband encoding (non-modulation) schemes. The backscatter communications in the RFID systems [7] usually adopt the FM0, a bi-phase space baseband encoding scheme. In the FM0, baseband phase is inverted at every symbol boundary, and a data-0 (i.e., bit 0 ) has an additional mid-symbol phase inversion, which is shown in Figure 1(a). The two sequences, permitted in the FM0 and each consisting of four symbols, are shown in Figure 1(b).

It was revealed in our previous study [31] that the ComBC is with the Energy Consumption Disparity (ECD). That is, backscattering data 0 (i.e., bit 0 ) expends much more energy than data 1 (i.e., bit 1). We refer to the bit with higher energy consumption as high energy bit (HEB) and the other as low energy bit (LEB). To use ECD, we presented the Energy-Efficient Data Delivery Scheme (EEDDS) [31] that delivers data via an energy-efficient codebook shared by the sender and the receiver. Under the EEDDS, the original bit stream in the sender is divided every $m$ bits to form $m$-bit data blocks and each block is backscattered to the receiver by using the designed codebook with $2^{m} n$-bit codewords, where $n$ stands for the length of a codeword. Surely, there exists one-to-one correspondence between the $m$-bit blocks and the codewords. The key to the EEDDS is that the designed codebook incorporates as many LEBs as possible so the sender can backscatter codewords energy-efficiently.

In the second half of 2018, we experienced difficulty in experimenting the EEDDS on Wireless Identification Sensing Platform (WISP) [21], which is embedded with the sensing, computing, and programmable hardware. This is because the EEDDS requires a big codebook to save energy. For example, the EEDDS can save energy only when $m>12$ in the case when the ECD is less than 2, and thus the codebook contains at least $2^{12}=4,096 n$-bit codewords. In other words, it needs a large memory space to hold the codebook. Unfortunately, the WISP only has totally $64 \mathrm{~KB}$ memory size to keep both programs and data. As a result, too large a codebook cannot squash into the small memory.

The above difficulty has been overcome by the prefix code based backscatter communications (CBBC) scheme, which we proposed in Reference [29]. In the CBBC, the original $m$-bit blocks are separated into two groups according to the number of HEBs in the blocks, and one of the groups is delivered with a prefix codebook while the other is backscattered directly without any codebook. Two different data rates are adopted by the sender to indicate whether a block is backscattered with its codeword or not.

The CBBC exhibits the drawback that it sacrifices throughput, i.e., it trades the throughput for energy saving. In other words, the $\mathrm{CBBC}$ brings a considerable throughput reduction compared with the ComBC although it can deliver more bits than the ComBC with the same energy. This encourages us to seek a better scheme so both energy consumption and throughput are considerably improved, which is the motivation of the article. 
The main contributions are as follows:

(1) We propose the dual-codebook based backscatter communications (DBBC) scheme. In addition, we formulate the optimization problem that minimizes the energy consumption over the wireless link between the sender and the receiver in the DBBC. After mapping a prefix codebook into a binary tree, we use pruning and expanding operation (PEO) to solve the optimization problem. The solution leads to the two energy-efficient codebooks and the optimal pair of parameters $m$ and $x$, where parameter $x$ is used in separating the original $m$-bit data blocks into two groups that correspond to the two codebooks, respectively. With the DBBC, the sender and the receiver share the two codebooks, and the sender delivers $m$ bits at a time by backscattering the codeword of the original $m$-bit data block. The sender adopts two different data rates to indicate which codebook is applied in delivering the ongoing data block.

(2) The experiments on WISP show that the DBBC can backscatter data $83 \%$ more than the ComBC without sacrificing throughput by using the solution of the formulated optimization problem.

The remainder of this article is organized as follows: Related works are surveyed in Section 2. We present the DBBC in Section 3, design the two prefix codebooks in Section 4, experiment on WISP to validate the energy saving with DBBC in Section 5, evaluate the performance of the DBBC via simulation in Section 6, and conclude the article in Section 7.

\section{RELATED WORK}

Energy harvesting technology has emerged as an alternative solution to energy limitation in the Internet of Things (IoT) [5]. In an energy-harvesting network, nodes can replenish energy through harvesting ambient renewable energy. The available energy sources include solar energy [23, 27], vibration energy [22], wind energy [3], radio frequency signals [31], and more.

Backscatter communications, a promising solution to green communication for future IoT [28], have been widely applied in delivering data in various wireless networks. V. Liu et al. [13] designed a backscatter communication system that enables two devices to communicate via leveraging existing TV and cellular transmissions. B. Kellogg et al. [11] considered Wi-Fi-backscatter and constructed communication links between an radio frequency (RF)-powered device and commercial Wi-Fi devices, in which the existing Wi-Fi infrastructure provides Internet connectivity for the RF-powered devices. D. Bharadia et al. [1] proposed a high-throughput communication system, called BackFi, which adopts ambient Wi-Fi transmissions as excitation signals to realize long-range communication between $\mathrm{Wi}-\mathrm{Fi}$ access points and IoT sensors backscattering with very low power. Iyer et al. [8] developed the intertechnology backscatter that makes a signal understood by the receivers operating with different protocols (e.g., Bluetooth backscatter signals are compatible with Wi-Fi and ZigBee signals), which promises the communications between Internet connected implanted devices. V. Talla et al. [24] designed the LoRa backscatter, whose range reaches $2.8 \mathrm{~km}$ when a backscatter device is close to the RF source transmitting with tens of microwatts of power, 1,000 times lower than the power of commercial LoRa radio chipsets. Y. Li [12] presented a Passive-ZigBee backscatter that can transform Wi-Fi packets into desired ZigBee ones with energy consumption of 1,440 times lower than commercial ZigBee devices. A. N. Parks et al. [19] introduced a multi-antenna cancellation design together with a coding mechanism, which supports long-range communication and concurrent transmissions for the backscatter devices. W. Gong et al. [4] presented a high-throughput rate adaptation scheme in which a rate mapping algorithm chooses the best rate for both downlink and uplink. B. Lyu et al. [14] considered the joint design of time allocation and reflection coefficient to maximize 
throughput in a typical backscatter communication system. J. Qian et al. [20] investigated the high-order modulation M-PSK to increase data rate of backscatter communication.

As for the aforementioned WISP, in addition to the compatibility with EPCglobal Gen2 Specification (i.e., an RFID protocol), it considerably extends the capability of RFID tags, because it contains the hardware that supports programming, sensing, computation, and storage [21]. WISP has been extensively applied. V. Talla and J. R. Smith [26] extended WISP and presented a hybrid analog-digital backscatter platform, which incorporates digital backscatter for control and addressability and analog backscatter for high data rate transmission. Y. Zhao et al. [30] designed NFC-WISP to support near field communication (NFC), and it can communicate with smart devices compatible with NFC. S. Naderiparizi et al. [16] developed the WISPCam (a battery-free RFID camera) to capture image and applied WISPCams to capture 3D location [17] and created battery-free high definition video streaming [15]. V. Talla et al. [25] developed a battery-free cellphone that harvests energy from RF signals and ambient light. In summary, WISP is extremely suitable for experimental platform to test and evaluate the considered backscatter communications schemes.

The FM0 baseband transmission is widely adopted in the RFID systems and WISP. As we revealed in Reference [31], the FM0 exhibits ECD in backscattering bit 0 (HEB) and bit 1 (LEB), because an additional mid-symbol inversion is needed in backscattering bit 0 , consuming much more energy than backscattering bit 1 . In view of the ECD, the EEDDS [31] reduces energy consumption in the backscatter communication by designing an energy-efficient codebook in which the codewords are of equal length. The codebook is shared by the sender and the receiver. Then, the sender just backscatters a codeword in the codebook instead of a bit block in the original bit stream, and the receiver recovers the original bit block by checking the shared codebook. The idea underlying the EEDDS is to accommodate as many LEBs in the codebook. The EEDDS has the shortcoming that it saves energy only when the designed codebook is relatively large, which defeats its implementation on WISP with small memory. This shortcoming is remedied by the PCBS [32], which adopts a prefix codebook containing the codewords with variable length. Under the EEDDS, the receiver cannot recover the original bit block until it receives an entire codeword. Under the PCBS, however, the receiver can try recovering the original bit block as soon as it receives a single bit, thanks to the prefix code, shortening data delivery delay. The CBBC introduced in Reference [29] achieves much energy efficiency than the PCBS and the EEDDS. Under the CBBC, the original $2^{m} m$-bit blocks are separated into two groups according to the number of HEBs in the blocks, and then the group with many HEBs is backscattered with a prefix codebook and the other group is backscattered without any codebook.

In this article, we present the $\mathrm{DBBC}$. The main differences between the $\mathrm{DBBC}$ and the previously published $\mathrm{CBBC}$ are as follows: First, the DBBC separates original $2^{m} m$-bit blocks into two groups and we design a prefix codebook for each group, whereas the $\mathrm{CBBC}$ only uses one codebook. Second, the DBBC gains more energy saving than the $\mathrm{CBBC}$ without sacrificing throughput, whereas the $\mathrm{CBBC}$ causes considerable throughput reduction.

To date, the research community makes much efforts on improving the communication efficiency of backscatter. The parallel backscatter techniques have emerged. J. Ou et al. [18] presented the BiGroup, an RFID communication paradigm allowing the reader to decode the collision from multiple commercial-off-the-shelf (COTS) RFID tags in one communication round to achieve multiple concurrent transmissions from COTS passive tags and improve the performance of physical layer collision recovery. P. Hu et al. [6] presented the LF-Backscatter, an asymmetric backscatter communication protocol enabling the nodes to blindly transmit data, in which powerful RFID readers are applied to detect the interleaving signal edges with high sampling rates and separate collided tag signals. M. Jin et al. [10] employed the highly stable probabilities of transitions between signals' combined states to decode the collided signals without relying on stable signals, 


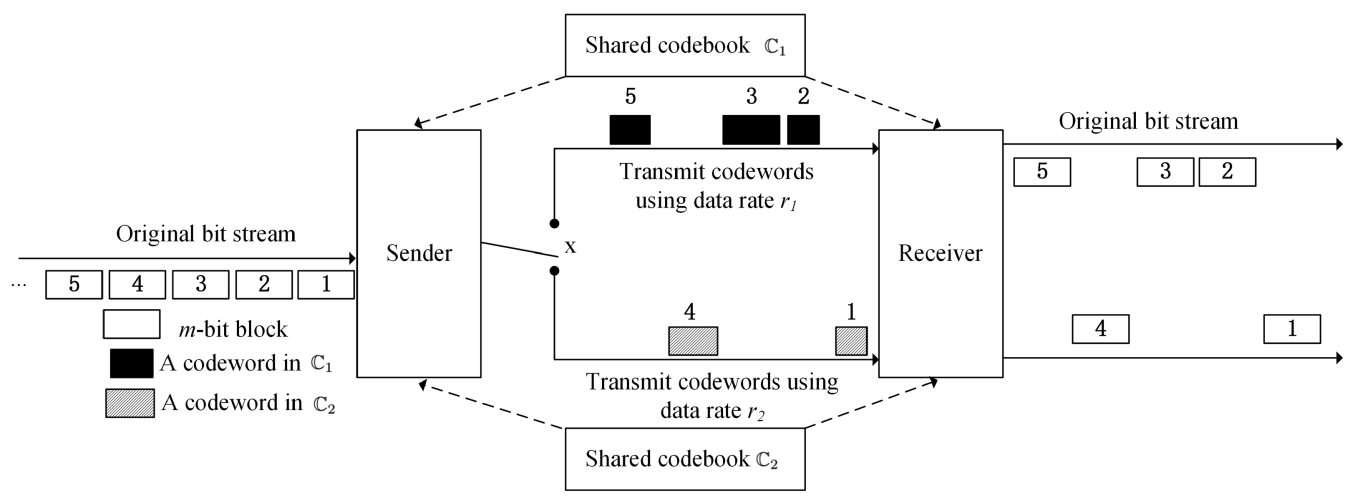

Fig. 2. Block diagram of the proposed DBBC.

which achieves highly reliable parallel decoding even in hostile channel conditions. M. Jin et al. [9] proposed the interstellar traveling model to capture the bursty Gaussian process of a collided signal and presented a reliable signal processing approach to support parallel backscatter in the wild. In the existing parallel backscatter techniques, the reader communicates with the tags, including the computational RFID (CRFID) tags (such as WISPs). The proposed DBBC can be applied in the CRFID tags. The reader and the CRFID tags share the two designed codebooks, and the CRFID tags backscatter codewords from which the reader recovers the original data using the codebooks.

\section{THE DBBC}

The proposed DBBC also adopts prefix code featured by the property that any codeword is not a prefix of any other codeword in a codebook [2].

Like the EEDDS [31] and the CBBC [29], the DBBC also delivers one $m$-bit data block after another by using codebook. Given $m$, there are totally $2^{m} m$-bit data blocks ranging from " $\underbrace{000 \ldots 00}_{m \text { bits }}$," “ $\underbrace{000 \ldots 01}, " \underbrace{000 \ldots 10}, \cdots, \cdots \underbrace{111 \ldots 11}$." Hence, we need to keep $2^{m}$ codewords in the memory if we only use a single codebook for backscattering $m$-bit data blocks, because it is required to have one-to-one correspondence between the $2^{m}$ blocks and the codewords in the codebook. As a result, the codebook size increases rapidly when $m$ grows, which may cause the designed codebook unable to be kept in the memory.

To fully keep the codewords in the memory, we adopt two codebooks in the DBBC, and these codebooks are shared by the sender and the receiver. Accordingly, the original $2^{m}$ data blocks are divided into two groups, which correspond to the two codebooks, respectively. For an $m$-bit data block, the sender backscatters its corresponding codeword rather than the original data block. We let the sender adopt two different data rates to inform the receiver of which codebook is applied so the receiver can recover the original $m$-bit data through the corresponding codebook.

The DBBC is illustrated in Figure 2, where the sender delivers data with the two shared codebooks $\mathbb{C}_{1}$ and $\mathbb{C}_{2}$ by using data rates $r_{1}$ and $r_{2}$. In the figure, data blocks 2,3 , and 5 are delivered with codebook $\mathbb{C}_{1}$ and data rate $r_{1}$, and blocks 1 and 4 are with codebook $\mathbb{C}_{2}$ and data rate $r_{2}$. The receiver recovers $m$ bits of the original data upon it receives a codeword by searching for the original $m$-bit block in the corresponding codebook judged by the applied data rate.

In addition to codebooks $\mathbb{C}_{1}$ and $\mathbb{C}_{2}$, the DBBC needs a pair of parameters $x$ and $m$. These codebooks and parameters are obtained through solving the optimization problem (12) formulated in Section 4.4. 
Table 1. Correspondence between Codewords and Data Blocks

\begin{tabular}{|c|c||c|c|}
\hline \multicolumn{2}{|c||}{$\mathbb{C}_{1}$} & \multicolumn{2}{c|}{$\mathbb{C}_{2}$} \\
\hline$m$-bit block & codeword & $m$-bit block & codeword \\
\hline 0000 & 000 & 1001 & 00 \\
0001 & 001 & 1010 & 010 \\
0010 & 010 & 1011 & 011 \\
0011 & 011 & 1100 & 100 \\
0100 & 100 & 1101 & 101 \\
0101 & 101 & 1110 & 110 \\
0110 & 110 & 1111 & 111 \\
0111 & 1110 & & \\
1000 & 1111 & & \\
\hline
\end{tabular}

The DBBC proceeds as follows:

Step 1. The sender and the receiver share $\mathbb{C}_{1}$ and $\mathbb{C}_{2}$.

Step 2 . The sender cuts the original data into blocks every $m$ bits and then divides the blocks into two groups $G_{1}=\{z: \operatorname{dec}(z)<x\}$ and $G_{2}=\{z: \operatorname{dec}(z) \geq x\}$, where $z$ stands for an $m$-bit block and $\operatorname{dec}(z)$ is the decimal number of $z$. Groups $G_{1}$ and $G_{2}$ correspond to $\mathbb{C}_{1}$ and $\mathbb{C}_{2}$, respectively. That is, each codeword in $\mathbb{C}_{i}$ maps exactly one $m$-bit block in $G_{i}$ and vice versa $(i=1,2)$.

Step 3. Before delivering an $m$-bit block, denoted by $z$, the sender searches in $\mathbb{C}_{1}$ for its corresponding codeword and then backscatters the codeword with data rate $r_{1}$ if $\operatorname{dec}(z)<x$, and it searches in $\mathbb{C}_{2}$ for the codeword and transmits it with data rate $r_{2}$ otherwise.

Step 4. Upon receiving a packet with data rate $r_{i}$, the receiver decodes the original $m$-bit data by using codebook $\mathbb{C}_{i}, i=1,2$.

To help the readers understand the DBBC, we use the following example in which $m=4$ and $x=9$. Assume the two codebooks are as listed in the Table 1 , where codebooks $\mathbb{C}_{1}$ and $\mathbb{C}_{2}$ have 9 and 7 codewords, respectively. In $\mathbb{C}_{1}$, each 4-bit block has decimal number less than $x$ (i.e., 9); and each 4-bit block in $\mathbb{C}_{2}$ has decimal number equal to or greater than 9 . Totally, there are 16 codewords that correspond to $2^{4} 4$-bit blocks. In either codebook, the left column lists the possible original 4-bit blocks while the right column holds the corresponding codewords.

Now, we elaborate how the DBBC brings in energy saving. Suppose occurrence of bit 0 and bit 1 in the original bit stream has the same probability. Thus, each of the $2^{m} m$-bit blocks has the same probability of occurring in the original data. Assume ECD is 2 . Thus, we can assume that backscattering LEB (i.e., bit 1) expends 1 unit of energy (UoE) and backscattering HEB (i.e., bit 0) consumes 2 UoEs [31]. There are 16 4-bit blocks that contain 32 LEBs and 32 HEBs in Table 1. In the case when the original blocks are backscattered directly without codebook, the total energy consumption of the original blocks is $32 \times(2+1)=96$ UoEs, and thus the average energy consumption per block is $96 / 16=6$ UoEs. Now, we consider the energy consumption in the proposed DBBC. In Table 1, codebook $\mathbb{C}_{1}$ contains 13 HEBs and 16 LEBs, which consumes $13 \times 2+16=42$ UoEs. Similarly, codebook $\mathbb{C}_{2}$ contains 9 HEBs and 11 LEBs and consumes $9 \times 2+11=29$ UoEs. As a result, the total energy consumption of the codewords in both codebooks is $42+29=71$ UoEs, which yields the average energy per codeword as $71 / 16=4.4375$ UoEs. Therefore, the proposed DBBC brings average energy saving of $6-4.4375=1.5625$ UoEs per 4-bit data block. 
It can be clearly seen from the above example that energy saving depends on parameters $m$ and $x$. In summary, the two codebooks and the two parameters are key to the DBBC. They will be addressed in the next section.

\section{CODEBOOK DESIGN}

As in Reference [29], we denote the $\operatorname{ECD}$ by $\alpha$, a constant greater than 1 , and assume

$$
\varepsilon_{0}=\alpha \varepsilon_{1},
$$

where $\varepsilon_{1}$ is the total energy consumption of the sender and the receiver in delivering one LEB (i.e., a bit 1 ) and $\varepsilon_{0}$ is that for a HEB (i.e., a bit 0 ).

For a given $m$, there are $2^{m}$ different $m$-bit data blocks. Among them, $x$ blocks are in $G_{1}=$ $\{z: \operatorname{dec}(z)<x\}$ and $2^{m}-x$ ones are in $G_{2}=\{z: \operatorname{dec}(z) \geq x\}$. Accordingly, codebooks $\mathbb{C}_{1}$ and $\mathbb{C}_{2}$ contain $x$ and $2^{m}-x$ codewords, respectively. That is, $\left|\mathbb{C}_{1}\right|=x$ and $\left|\mathbb{C}_{2}\right|=2^{m}-x$, where notation "| | " to represent the cardinality of a set.

Assume the probabilities of LEB and HEB occuring in the original data stream are identical, which will be referred to as "identical probability assumption" in the sequel. Then, the event that an $m$-bit block corresponds to a codeword in $\mathbb{C}_{1}$ has the probability

$$
P_{1}=\frac{\left|\mathbb{C}_{1}\right|}{2^{m}}=\frac{x}{2^{m}}
$$

and the event that an $m$-bit block corresponds to a codeword in $\mathbb{C}_{2}$ has probability

$$
P_{2}=\frac{\left|\mathbb{C}_{2}\right|}{2^{m}}=1-\frac{x}{2^{m}}
$$

\subsection{Energy Consumption of The ComBC}

When the original $m$-bit blocks are backscattered by the ComBC, which does not use any codebook, the $2^{m} m$-bit blocks have totally $2^{m} m$ bits, where LEBs and HEBs are each $m 2^{m-1}$. Therefore, under the identical probability assumption, we have the average energy consumption of delivering one $m$-bit block from the sender to the receiver under the ComBC as follows [29]:

$$
E_{c b c}=\frac{m 2^{m-1} \varepsilon_{0}+m 2^{m-1} \varepsilon_{1}}{2^{m}}=\frac{1}{2} m(1+\alpha) \varepsilon_{1} .
$$

\subsection{Energy Consumption of the DBBC}

Now, we study the energy consumption in the DBBC. For codebook $\mathbb{C}_{1}$ with $x$ elements, the codewords in $\mathbb{C}_{1}$ can be represented by $c^{(i)}=b_{1}^{(i)} b_{2}^{(i)} \cdots b_{u_{i}}^{(i)}$, where $b_{j}^{(i)}\left(j=1,2, \ldots, u_{i}\right)$ stands for a bit, and $u_{i}$ is the length of $c^{(i)}, i \in I_{1}=\{1,2, \ldots, x\}$. Thus, the number of bit $1 \mathrm{~s}$ in codeword $c^{(i)}$ is $n_{1}^{(i)}=\sum_{j=1}^{u_{i}} b_{j}^{(i)}$ and that of bit $0 \mathrm{~s}$ is $n_{0}^{(i)}=u_{i}-\sum_{j=1}^{u_{i}} b_{j}^{(i)}$. As a result, the total energy consumption in delivering $c^{(i)}$ over the wireless link between the sender and the receiver is $\varepsilon_{1} n_{1}^{(i)}+\varepsilon_{0} n_{0}^{(i)}=$ $\varepsilon_{1}\left[\alpha u_{i}+(1-\alpha) \sum_{j=1}^{u_{i}} b_{j}^{(i)}\right]$. This yields the average energy consumption per codeword in $\mathbb{C}_{1}$ as

$$
E_{1}=\frac{1}{\left|\mathbb{C}_{1}\right|} \sum_{c^{(i)} \in \mathbb{C}_{1}} \varepsilon_{1}\left[\alpha u_{i}+(1-\alpha) \sum_{j=1}^{u_{i}} b_{j}^{(i)}\right]=\frac{\varepsilon_{1}}{x} \sum_{i=1}^{x}\left[\alpha u_{i}+(1-\alpha) \sum_{j=1}^{u_{i}} b_{j}^{(i)}\right] .
$$

Similarly, we express a codeword in $\mathbb{C}_{2}$ with $2^{m}-x$ elements as $d^{(i)}=g_{1}^{(i)} g_{2}^{(i)} \ldots g_{v_{i}}^{(i)}$, where $g_{j}^{(i)}\left(j=1,2, \ldots, v_{i}\right)$ stands for a bit, $v_{i}$ is the codeword's length, and $i \in I_{2}=\left\{1,2, \ldots, 2^{m}-x\right\}$. The number of bit $1 \mathrm{~s}$ and bit $0 \mathrm{~s}$ in codeword $d^{(i)}$ are $\sum_{j=1}^{v_{i}} g_{j}^{(i)}$ and $v_{i}-\sum_{j=1}^{v_{i}} g_{j}^{(i)}$, respectively. Thus, 
the energy consumption of delivering $d^{(i)}$ is $\varepsilon_{1}\left[\alpha v_{i}+(1-\alpha) \sum_{j=1}^{v_{i}} g_{j}^{(i)}\right]$, and the average energy consumption over the wireless link per codeword in $\mathbb{C}_{2}$ is

$$
E_{2}=\frac{\varepsilon_{1}}{2^{m}-x} \sum_{i=1}^{2^{m}-x}\left[\alpha v_{i}+(1-\alpha) \sum_{j=1}^{v_{i}} g_{j}^{(i)}\right]
$$

Therefore, under the identical probability assumption, the expected energy consumption in delivering one $m$-bit data block over the wireless link between the sender and the receiver in the DBBC with codebooks $\mathbb{C}_{1}$ and $\mathbb{C}_{2}$ is

$$
E_{d b b c}=P_{1} E_{1}+P_{2} E_{2}=\frac{\varepsilon_{1}}{2^{m}}\left\{\sum_{i=1}^{x}\left[\alpha u_{i}+(1-\alpha) \sum_{j=1}^{u_{i}} b_{j}^{(i)}\right]+\sum_{i=1}^{2^{m}-x}\left[\alpha v_{i}+(1-\alpha) \sum_{j=1}^{v_{i}} g_{j}^{(i)}\right]\right\} .
$$

Clearly, to deliver more bits than the ComBC with the same energy, we desire to meet the following equation in designing the two codebooks used in the DBBC:

$$
E_{d b b c}<E_{c b c} .
$$

\subsection{Bit Duration in the DBBC}

Under the DBBC, an $m$-bit block in $G_{i}$ uniquely corresponds to one codeword in $\mathbb{C}_{1} \cup \mathbb{C}_{2}$. It takes time of $u_{i} / r_{1}$ to backscatter codeword $c^{(i)} \in \mathbb{C}_{1}$, where $r_{1}$ is the data rate applied in delivering the codeword. This leads to the average delivery time per codeword in $\mathbb{C}_{1}$ as $\frac{1}{x} \sum_{i=1}^{x} \frac{u_{i}}{r_{1}}$, which is equivalent to the duration of delivering an $m$-bit block. Thus, we obtain the average delivery time per bit, simply referred to as "bit duration" below, for the data blocks in $G_{1}$ as

$$
t_{b, 1}=\frac{1}{m x} \sum_{i=1}^{x} \frac{u_{i}}{r_{1}}
$$

Similarly, the time of transmitting codeword $d^{(i)} \in \mathbb{C}_{2}$ is $v_{i} / r_{2}$. Then, we obtain the average delivery time per codeword in $\mathbb{C}_{2}$ as $\frac{1}{2^{m}-x} \sum_{i=1}^{2^{m}-x} \frac{v_{i}}{r_{2}}$, which represents the duration of delivering an $m$-bit block. Thus, bit duration for the data blocks in $G_{2}$ is

$$
t_{b, 2}=\frac{1}{m\left(2^{m}-x\right)} \sum_{i=1}^{2^{m}-x} \frac{v_{i}}{r_{2}}
$$

Therefore, the expected bit duration in the $\mathrm{DBBC}$ is

$$
t_{b}=P_{1} t_{b, 1}+P_{2} t_{b, 2}=\frac{1}{m 2^{m}}\left[\frac{1}{r_{1}} \sum_{i=1}^{x} u_{i}+\frac{1}{r_{2}} \sum_{i=1}^{2^{m}-x} v_{i}\right]
$$




\subsection{The Optimization Problem}

We use the optimization problem in Equation (12) to find the optimal parameters $x$ and $m$ and the optimal prefix codebooks $\mathbb{C}_{1}$ and $\mathbb{C}_{2}$ for our DBBC.

$$
\begin{aligned}
& \min E_{d b b c} \\
& \text { w.r.t. : } m, x, b_{j}^{(i)}\left(i=1,2, \ldots, x ; j=1,2, \ldots, u_{i}\right), \\
& g_{j}^{(i)}\left(i=1,2, \ldots, 2^{m}-x ; j=1,2, \ldots, v_{i}\right) \\
& \text { s.t. : } \sum_{i=1}^{x}\left[\alpha u_{i}+(1-\alpha) \sum_{j=1}^{u_{i}} b_{j}^{(i)}\right] \\
& +\sum_{i=1}^{2^{m}-x}\left[\alpha v_{i}+(1-\alpha) \sum_{j=1}^{v_{i}} g_{j}^{(i)}\right]<2^{m-1} m(1+\alpha) ; \\
& \sum_{i, j}^{c_{i, j}} b_{k}^{(i)} \oplus b_{k}^{(j)} \neq 0, \forall i, j \in\{1,2, \ldots, x\}(i \neq j) ; \\
& \sum_{i=1}^{x} \frac{1}{2^{u_{i}}} \leq 1 ; \\
& \sum_{i, j} g_{k}^{(i)} \oplus g_{k}^{(j)} \neq 0, \forall i, j \in\left\{1,2, \ldots, 2^{m}-x\right\}(i \neq j) ; \\
& k_{k=1}^{2_{-x}} \sum_{i=1}^{1} \frac{1}{2^{v_{i}}} \leq 1 ; \\
& \frac{1}{m 2^{m}}\left[\frac{1}{r_{1}} \sum_{i=1}^{x} u_{i}+\frac{1}{r_{2}} \sum_{i=1}^{2^{m}-x} v_{i}\right] \leq \frac{\theta}{\max \left\{r_{1}, r_{2}\right\}} ; \\
& 2 \leq m \leq m ; \\
& b_{j}^{(i)} \in\{0,1\}, i=1,2, \ldots, x ; j=1,2, \ldots, u_{i} ; \\
& g_{j}^{(i)} \in\{0,1\}, i=1,2, \ldots, 2^{m}-x ; j=1,2, \ldots, v_{i} ; \\
& x \in\left\{1,2, \ldots, 2^{m}-1\right\} .
\end{aligned}
$$

In Equation (12), notation “ $\oplus$ ” stands for the bitwise exclusive-OR operation. Constraint (12)(a) is the same as Equation (8) into which Equations (4) and (7) are substituted. Equations (12)(b) and (12)(d) reflect prefix property, where $\underline{c}_{i, j}=\min \left\{u_{i}, u_{j}\right\}$ and $\underline{d}_{i, j}=\min \left\{v_{i}, v_{j}\right\}$. Equations (12)(c) and (12)(e) are the Kraft inequalities. Equations (12)(b), (12)(c), (12)(d), and (12)(e) are required in designing prefix code [2]. In Equation (12)(f), $\theta$ is a positive constant, $r=\max \left\{r_{2}, r_{1}\right\}$, and $1 / r$ represents the bit duration when the higher rate in $r_{1}$ and $r_{2}$ is applied. As a result, Constraint (12)(f) aims to limit bit duration $t_{b}$ within $\theta$ multiples of the duration $1 / r$. To accelerate data delivery, we let the sender backscatter the codebook with more bits by applying the higher data rate. In other words, we set $r_{1}=r$ if $\sum_{i=1}^{x} u_{i}>\sum_{i=1}^{2^{m}-x} v_{i}$ (i.e., the number of bits in the $\mathbb{C}_{1}$ is larger than that in the $\mathbb{C}_{2}$ ), and $r_{2}=r$ otherwise. In Equation $(12)(\mathrm{g}), \hat{m}$ is a preset constant used to limit the sizes of the two codebooks so the derived codebooks can be easily kept in small memory spaces of the nodes. 


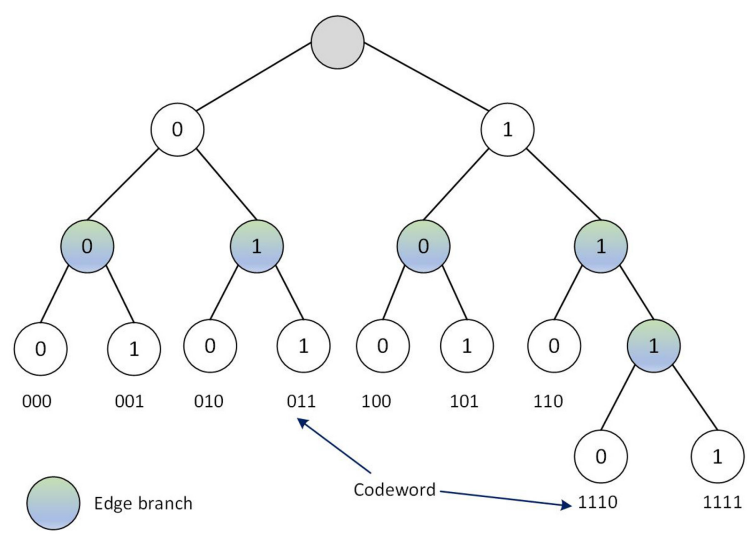

(a) The binary tree for codebook $\mathbb{C}_{1}$

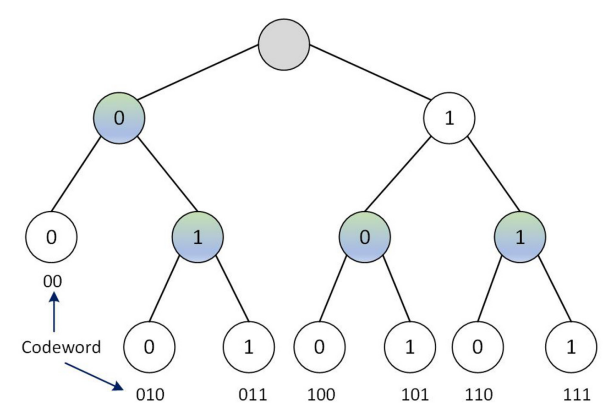

(b) The binary tree for codebook $\mathbb{C}_{2}$

Fig. 3. Binary tree presentation of prefix codebook.

\subsection{Solution of the Optimization Problem}

As we did in Reference [2], we use binary tree to represent prefix codebook and then perform pruning and expanding operation (PEO) on binary trees to find the solution of the optimization problem in (12).

In a binary tree, for each node with children, its left child is labelled by "0" and its right child by "1." Each leaf corresponds to the codeword composed of the bit string from the root to the leaf. For example, codebooks $\mathbb{C}_{1}$ and $\mathbb{C}_{2}$ in Table 1 are expressed as the trees in Figures 3(a) and 3(b), respectively.

Given a binary tree, we refer to a branch of the tree as an edge branch if the branch has at least one leaf as its child. For instance, the edge branches are with shadow in Figure 3.

PEO affects a pair of nodes, referred to as a PEO-pair below, in a binary tree. With PEO, the pruning operation chooses one of the edge branches to remove its leaf child/children while the expanding operation chooses a leaf to make it an edge branch that exactly has two children labeled by " 0 " and " 1 ," respectively. The edge branch for pruning operation and the leaf for expanding operation are a PEO-pair.

We give the following example to illustrate a PEO on the binary tree in Figure 3(b), which corresponds to codebook $\mathbb{C}_{2}=\{00,010,011,100,101,110,111\}$. Assume the PEO intends to prune leaf children of edge branch A and expands leaf B as shown in Figure 4(a), i.e., the two children of edge branch $\mathrm{A}$ are removed, and leaf $\mathrm{B}$ is expanded. Then, the $\mathrm{PEO}$ leads to the binary tree as shown in Figure 4(b), which corresponds to codebook $\tilde{\mathbb{C}}_{2}=\{00,01,100,101,1100,1101,111\}$. Therefore, the PEO changes codebook $\mathbb{C}_{2}$ into $\tilde{\mathbb{C}}_{2}$. It should be pointed out that it is possible to choose an edge branch with a single leaf and remove the only leaf (e.g., the leftmost edge branch in Figure 4(a)). In this case, only one leaf is removed by pruning operation, but the expanding operation still adds two leaves to the leaf to be expanded. The reason is that PEO keeps the number of leaves unchanged so the number of codewords remains.

Clearly, different PEOs lead to different binary trees, which correspond to different codebooks. To find the energy-efficient codebooks, we need to define weight of a codebook. Considering ECD represents the energy consumption ratio of a HEB to a LEB, we assume an LEB and an HEB consume 1 and $\alpha$ UoEs, respectively. Then, for a codeword with $s_{1}$ LEBs and $s_{0}$ HEBs, we define its weight as the sum $s_{1}+s_{0} \alpha$ because its LEBs and HEBs consume energy of $s_{1}$ and $s_{0} \alpha$ UoEs, respectively. Moreover, we define the weight of a codebook as the sum of the weights of the codewords in the codebook. 


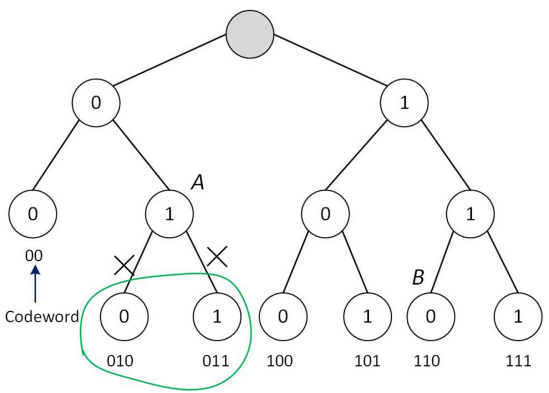

(a) The children of edge branch $\mathrm{A}$ to be pruned

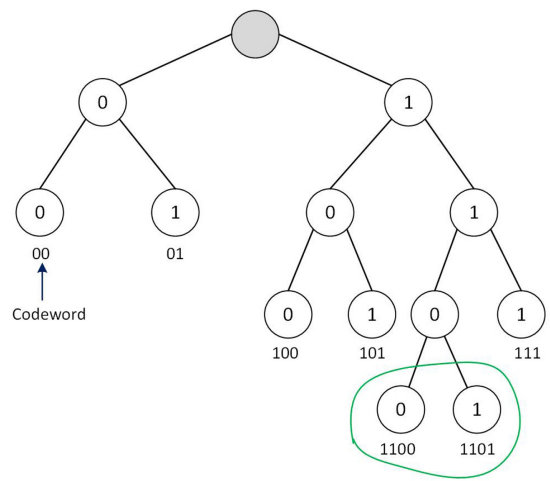

(b) Leaf B is expanded

Fig. 4. PEO.

For example, in Figure 4, codebook $\mathbb{C}_{2}$, which corresponds to the binary tree in Figure 4(a), has weight of $11+9 \alpha$, as there are 11 LEBs (bit 1 s) and 9 HEBs (bit 0 s) in the codewords with each corresponding to a leaf in the tree. Similarly, codebook $\tilde{\mathbb{C}}_{2}$, which corresponds to the binary tree in Figure 4(b), has weight of $12+9 \alpha$. As a result, codebook $\tilde{\mathbb{C}}_{2}$ has a greater weight than codebook $\mathbb{C}_{2}$. Equivalently, $\tilde{\mathbb{C}}_{2}$ consumes more energy than codebook $\mathbb{C}_{2}$. We prefer $\mathbb{C}_{2}$ to $\tilde{\mathbb{C}}_{2}$. Therefore, $\mathrm{PEO}$ strives to search such a PEO-pair that the codebook resulting from the $\mathrm{PEO}$ has a lower weight than the codebook corresponding to the binary tree before the PEO.

In the proposed DBBC, there are two codebooks, which correspond to two binary trees. We adopt a heuristic algorithm to find the two energy-efficient trees. Given binary tree $T_{i}$, let $A_{i}$ be the edge branch with child/children to be pruned and $B_{i}$ the leaf node to be expanded by the $\operatorname{PEO}(i=1,2)$. Let $T_{i}^{\prime}$ stand for the binary tree resulting from the PEO on $T_{i}(i=1,2)$. We use $w(T)$ to represent the weight of the codebook that corresponds to binary tree $T$. Then, the weight variation resulting from the PEO can be expressed as

$$
\delta\left(T_{i}, T_{i}^{\prime}\right)=w\left(T_{i}\right)-w\left(T_{i}^{\prime}\right),
$$

where the $w\left(T_{i}\right)$ stands for the weight of the tree $T_{i}$ prior to the PEO while the $w\left(T_{i}^{\prime}\right)$ for that of the tree $T_{i}^{\prime}$ generated by the PEO $(i=1,2)$. We prefer the PEO that results in a positive weight variation, i.e., $\delta\left(T_{i}, T_{i}^{\prime}\right)>0$, which indicates the generated tree $T_{i}^{\prime}$ having a lower energy consumption $(i=1,2)$. Hence, we need an algorithm to find the best PEO-pair so the PEO on the pair can gain the highest weight variation $\delta(\cdot)$.

Before presenting the algorithm, we introduce some notations. Let $e_{1}\left(T_{1}, T_{2}, m, \alpha\right)$ be the energy consumption per bit in the $\mathrm{DBBC}$, where $\alpha$ and $m$ are, respectively, the ECD and the length of original data block, and $T_{1}$ and $T_{2}$ are the binary trees corresponding to the two codebooks used in the DBBC. From Equation (4), we have the average energy consumption per bit in the ComBC as $\frac{1}{2}(1+\alpha) \varepsilon_{1}$. Compared with the ComBC, the "percentage of energy saving (PoES)" in the DBBC can be calculated by

$$
\xi\left(T_{1}, T_{2}, m, \alpha\right)=\frac{\frac{1}{2}(1+\alpha) \varepsilon_{1}-e_{1}\left(T_{1}, T_{2}, m, \alpha\right)}{\frac{1}{2}(1+\alpha) \varepsilon_{1}}=1-\frac{2 e_{1}\left(T_{1}, T_{2}, m, \alpha\right)}{(1+\alpha) \varepsilon_{1}} .
$$

In addition, we introduce notation

$$
\zeta\left(T_{1}, T_{2}, r_{1}, r_{2}\right)=\frac{\max \left\{r_{1}, r_{2}\right\}}{m 2^{m}}\left[\frac{1}{r_{1}} \sum_{i=1}^{x} u_{i}+\frac{1}{r_{2}} \sum_{i=1}^{2^{m}-x} v_{i}\right] .
$$


Then, Constraint (12)(f) of the optimization problem in Equation (12) turns into

$$
\zeta\left(T_{1}, T_{2}, r_{1}, r_{2}\right) \leq \theta .
$$

The optimization problem in Equation (12) is solved by Algorithm 1, where child $(A)$ represents the set of leaf children of node $A$. In the algorithm, the for loop in statements $2-19$ finds the best pair of parameters $m$ and $x$, where $x$ is used to separate the original $m$-bit blocks into two
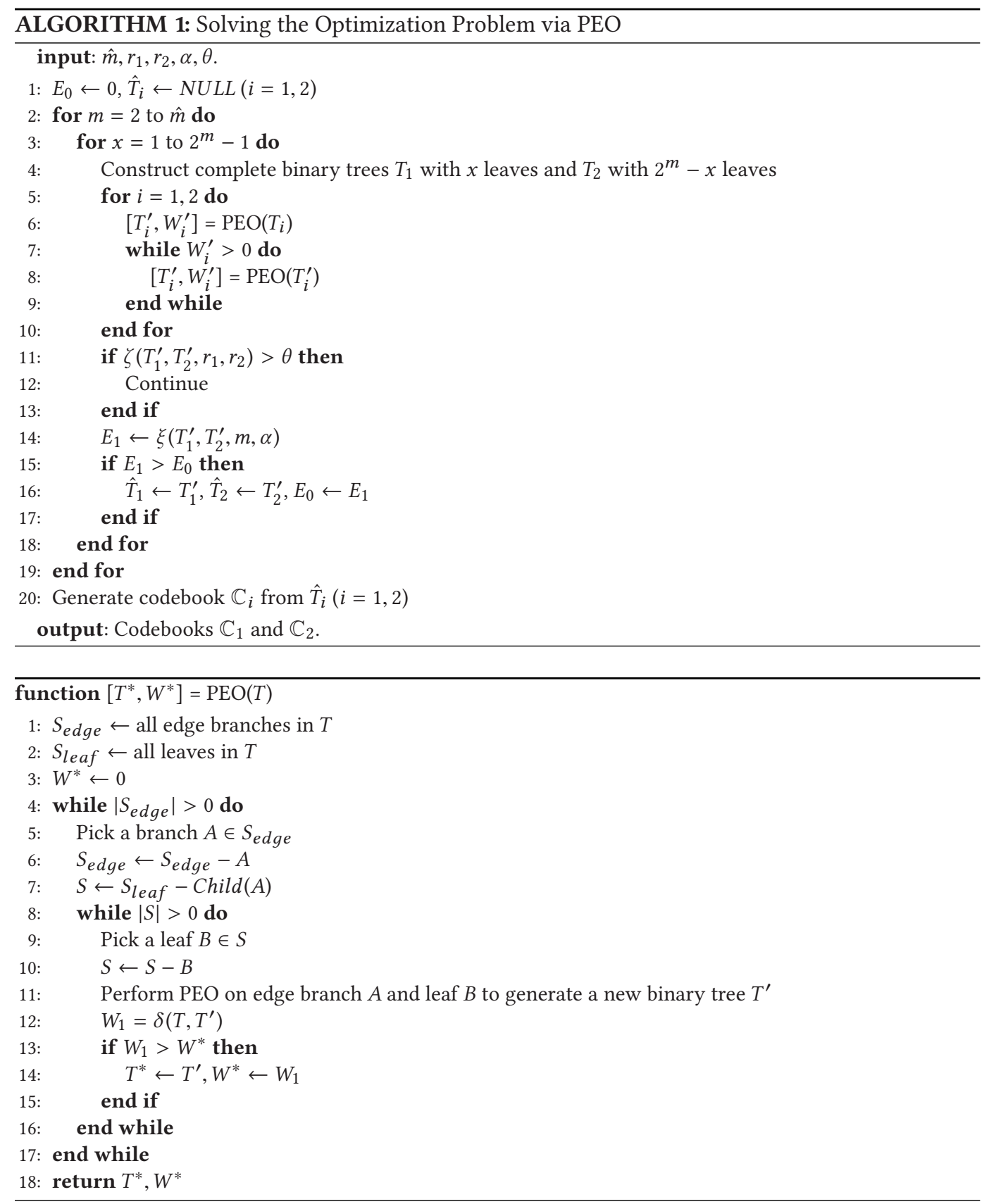
groups. Statements $5-10$ find the binary tree $T_{i}^{\prime}$ with the best PoES by iterative PEO on binary tree initiated from $T_{i}(i=1,2)$. Statements $11-13$ check whether Constraint (12)(f) of optimization problem (12) is satisfied or not, and only the pair of trees $T_{1}$ and $T_{2}$ satisfying the constraint go to the next statement for calculating the PoES. In function PEO(·), statements $4-17$ consider all the PEO-pairs with each consisting of an edge branch and a leaf in tree $T$ to yield a new tree $T^{\prime}$ and seek the binary tree that has the maximum weight variation $\delta(\cdot)$ through statements $12-15$.

Next, we analyze the complexity of Algorithm 1 . Let $m_{i, 1}$ and $m_{i, 2}$ be the number of edge branches and that of leaves in $T_{i}$, respectively $(i=1,2)$. Clearly, given a binary tree, the number of its edge branches is usually no more than that of the leaves. Noticing that there are totally $2^{m}$ leaves in trees $T_{1}$ and $T_{2}$, we have

$$
m_{i, 1}+m_{i, 2} \leq 2^{m}(i=1,2) .
$$

Statements $4-17$ in function $\mathrm{PEO}(\cdot)$ perform $m_{i, 1} m_{i, 2}$ times of PEOs, and thus the times of PEOs conducted on $T_{i}$ is

$$
N_{P E O}=m_{i, 1} m_{i, 2} \leq\left(\frac{m_{i, 1}+m_{i, 2}}{2}\right)^{2} \leq 4^{m-1},
$$

where Equation (16) is applied.

In the full binary tree with $2^{m}$ leaves, the number of bit $1 \mathrm{~s}$ and that of bit $0 \mathrm{~s}$ are identical, and the leaves correspond to the codewords: " $\underbrace{000 \ldots 00}_{m \text { bits }}, " \underbrace{000 \ldots 01}$, " $\underbrace{000 \ldots 10}, \cdots$ and " $\underbrace{111 \ldots 11}$. As a result, the codebook holding these codewords has the same numbers of bit $1 \mathrm{~s}$ and bit $0 \mathrm{~s}$ as $\left(m 2^{m}\right) / 2$, and thus the codebook has weight of $m 2^{m-1}(1+\alpha)$. The initial binary tree $T_{i}$ in Algorithm 1 is a complete tree; it has the weight less than the full binary tree. Hence, we have

$$
w\left(T_{i}\right)<m 2^{m-1}(1+\alpha) .
$$

In the algorithm, each time of executing function $\mathrm{PEO}(\cdot)$ reduces the weight of tree $T_{i}$ by at least $\alpha-1$ UoEs. Thus, the while loop in statements $7-9$ in Algorithm 1 calls function $\mathrm{PEO}(\cdot)$ no more than $\frac{w\left(T_{i}\right)}{\alpha-1}$ times. In other words, the while loop conducts PEO on $T_{i}$ for the times as follows:

$$
\boldsymbol{\aleph}\left(T_{i}\right)=\frac{w\left(T_{i}\right)}{\alpha-1} N_{P E O}<\frac{\alpha+1}{\alpha-1} m 2^{3 m-3}(i=1,2),
$$

where Equations (17) and (18) are applied. In addition, the for loop in statements $5-10$ in Algorithm 1 conducts $\mathrm{PEO}$ on both $T_{1}$ and $T_{2}$, and thus it has the times of PEOs as $\boldsymbol{\aleph}\left(T_{1}\right)+\boldsymbol{\aleph}\left(T_{2}\right)<$ $\frac{\alpha+1}{\alpha-1} m 2^{3 m-2}$. Statements $3-18$ in Algorithm 1 generate the optimal trees $T_{1}^{\prime}$ and $T_{2}^{\prime}$ for $2^{m}-1$ times, which perform PEO for the times less than $\left(2^{m}-1\right)\left(\boldsymbol{\aleph}\left(T_{1}\right)+\boldsymbol{\aleph}\left(T_{2}\right)\right)<\frac{\alpha+1}{\alpha-1} m 2^{4 m-2}$ for a given $m$. Considering the outermost for loop in statements $2-19$ in Algorithm 1, the total number of PEOs conducted in the algorithm is no more than

$$
\boldsymbol{\aleph}=\sum_{m=2}^{\hat{m}} \frac{\alpha+1}{\alpha-1} m 2^{4 m-2}=\frac{\alpha+1}{60(\alpha-1)}\left[\left(\hat{m}-\frac{1}{15}\right) 16^{\hat{m}+1}-\frac{3584}{15}\right] .
$$

It should be pointed out that we can solve the optimization problem offline via Algorithm 1 . Hence, the complexity of the algorithm is acceptable, because $\hat{m}$ is quite small due to the fact that we need to put the designed two codebooks in small memory space of WISP. For the case when the nodes have sufficient memory space to store large sizes of the codebooks, we can apply Genetic Algorithm in solving the optimization problem (please refer to Reference [32]). 


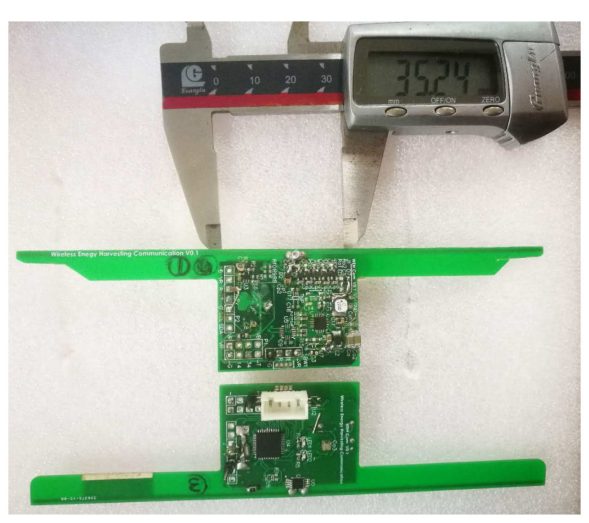

(a) The front and the back sides of a WISP

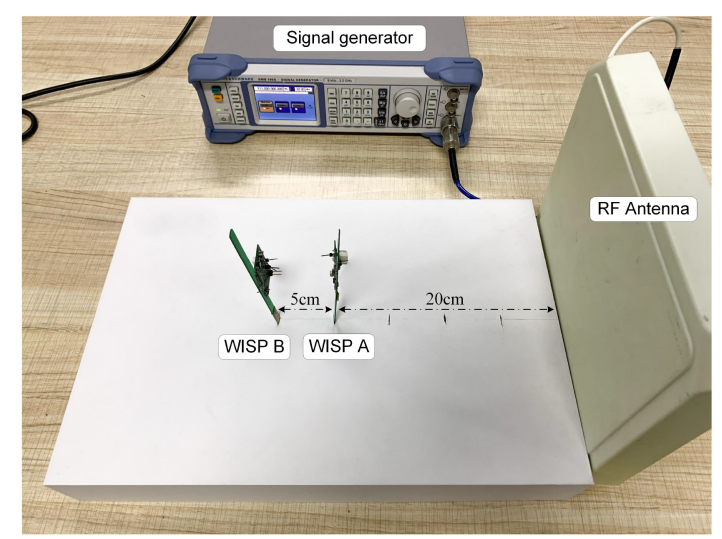

(b) Experiment on WISP

Fig. 5. Experiment on WISP.

\section{EXPERIMENT ON WISP}

In this section, we conduct experiment to evaluate the performance of the proposed DBBC. Considering WISP supports backscatter communications compatible with the RFID protocol and can be programmed [21] to implement the DBBC, we choose a pair of WISPs, called WISP A and WISP $\mathrm{B}$ below, to test the DBBC's performance. Each WISP has a micro-controller unit (MCU), $64 \mathrm{~KB}$ memory space, and a capacity with volume of $400 \mu \mathrm{F}$ (the capacity is used in storing the harvested energy). The front and the back sides of one of the WISPs are shown in Figure 5(a), where the side length of the square in the circuit board is around $36 \mathrm{~mm}$. We fabricate the two WISPs with each consisting of the antenna whose front end follows the WISP version 4, the MCU (i.e., MSP430FR5969) following the WISP version 5, and the BQ25570 chip with function of energy harvesting and power management. Here, WISP version 4 and WISP version 5 were published by the University of Washington, USA. Moreover, we develop the firmware to accomplish communications between the fabricated WISPs. In the experiment, we need to design two small codebooks such that each WISP can store the two codebooks in its small memory and deliver data based on the codebooks.

The experimental scenario is shown in Figure 5(b), where the WISPs A and B harvest energy from the radio signals from the antenna attached to the ROHDE \& SCHWARZ signal generator with model SMB100, and WISP A backscatters its data to WISP B. In fact, the distance between the RF antenna and a WISP affects the energy harvested by the WISP. In addition, the distance between the two WISPs may affect the bit error ratio (BER), i.e., it may impact how many bits are correctly received by WISP $\mathrm{B}$. Considering this work focuses on increasing the number of bits transmitted by the sender (i.e., WISP A) with the same energy as the ComBC, we ignore whether the transmitted bits are correctly received by WISP B or not currently and will deeply investigate the BER-related problem in the future.

As pointed out in our earlier work [31], we could not find an instrument to directly measure the amount of energy consumption of a WISP like the way of measuring circuit voltage or current with a multimeter, because the multimeter itself consumes considerable energy compared with the energy harvested by the WISP. Thus, we turn to the alternate means that monitors the energy consumption during the "peak-to-nadir period," which is defined as the interval from the instant when WISP A is full of energy to the instant when it exhausts its energy. In fact, each WISP used in the experiment is fully charged when the voltage of its capacity reaches $4.25 \mathrm{~V}$, called "the peak 
voltage," and it exhausts energy when the voltage drops to $2.0 \mathrm{~V}$, called "the nadir voltage." We believe that the WISP expends the close energy in every peak-to-nadir period, and thus, we focus on how many bits are transmitted by WISP A in a peak-to-nadir period.

Initially, the signal generator continuously transmits steady radio frequency signals, from which the WISPs harvest energy (the WISPs transfer the radio frequency signals to current), till both WISPs are fully charged. A peak-to-nadir period proceeds as follows: Upon having the peak voltage, WISP A starts continuous backscattering data with its MCU working at voltage above $2.0 \mathrm{~V}$. The WISP stops transmitting but harvests energy again when its capacity reaches the nadir voltage. The above peak-to-nadir process repeats as soon as WISP A is fully charged, which is judged by the peak voltage.

From the optimization problem in Equation (12), we need to determine $\alpha$, which reflects the ECD between delivering (or backscattering) HEB and LEB, before deriving the energy-efficient codebooks used in our DBBC in the experiment. To estimate $\alpha$, we perform the following twostep procedure on a WISP. In the first step, the WISP is fully charged and then transmits bit $1 \mathrm{~s}$ repetitively until the WISP exhausts its energy, which yields the number of the transmitted bit 1 $s$ during the peak-to-nadir period, denoted by $n_{1}$. In the second step, the WISP is fully charged and then transmits bit $0 \mathrm{~s}$ repetitively till the energy is depleted, which generates the number of the transmitted bit 0 , denoted by $n_{2}$. Then, the ECD $\alpha$ is estimated by $n_{1} / n_{2}$, where $n_{1}>n_{2}$, since backscattering bit 0 consumes more energy than backscattering bit 1 in the WISP. We obtain $\alpha=1.64$ over the average of 10 experiments. As a result, we set $\varepsilon_{1}=1 \mathrm{UoE}$ and $\varepsilon_{0}=1.64 \mathrm{UoEs}$ in deriving the two codebooks through the optimization problem (12) for the proposed DBBC. Moreover, we set $\hat{m}=12$ for Constraint (12)(g).

Next, we compare the DBBC with the previous CBBC [29] via experiment on WISP. The comparison is based on the metrics of "percentage of increased number of transmitted bits (PoINTB)" and "percentage of reduced throughput (PoRT)," which are defined in Reference [29]. The PoINTB is defined as $\left(a_{1}-a_{2}\right) / a_{2}$, where $a_{2}$ stands for the number of backscattered bits under the ComBC and $a_{1}$ is that under the DBBC or CBBC. Additionally, the PoRT is the ratio of $\left(b_{1}-b_{2}\right) / b_{1}$, where $b_{1}$ is the throughput of the ComBC and $b_{2}$ is the throughput of the $\mathrm{DBBC}$ or CBBC. Obviously, both metrics adopt the ComBC as the baseline.

We notice that two data rates of 3 and $1 \mathrm{kbps}$ are applied in the $\mathrm{CBBC}$, i.e., $r_{1}, r_{2} \in\{1,3\} \mathrm{kbps}$ [29]. We apply the same data rates in the proposed DBBC. Moreover, for the fair comparison, we let the ComBC backscatter data with the maximum data rate $r=3 \mathrm{kbps}$. The backscatter communications in these schemes adopt the bi-phase FM0 baseband encoding, in which each bit 0 has an additional mid-symbol phase inversion in addition to the phase inversion at its symbol boundary. Arrival of each phase inversion generates an interruption signal at the receiver (i.e., WISP B). Hence, the intervals of successive interruption signals can be classified as the "long interval" and the "short interval." The long interval, denoted by $T_{1}$, is the interval of two successive symbol boundaries; and the short interval, denoted by $T_{0}$, is the interval between a symbol boundary and the instant at which a mid-symbol phase inversion occurs. It holds that $T_{1}=2 T_{0}$. With the CBBC or the DBBC, the receiver recovers a bit 1 if a long interval is captured, and it recovers a bit 0 if two successive short intervals are captured. Given a pair of data rates $r_{1}$ and $r_{2}$, let $T_{i, 1}$ and $T_{i, 0}$ be the long interval and the short interval, respectively, for data rate $r_{i}(i=1,2)$. Then, we have $T_{1,1}=2 T_{1,0}$ and $T_{2,1}=$ $2 T_{2,0}$. Without loss of generality, we assume $T_{1,1}>T_{2,1}$. As a result, we should choose a pair of data rates $r_{1}$ and $r_{2}$ such that $T_{2,1}<T_{1,0}$, which enables the receiver to discriminate the two data rates. This is why we set one data rate to be 3 times of the other for a pair of data rates.

The experimental results given below are the average of 20 experiments. In other words, for each of the ComBC, the $\mathrm{CBBC}$, and the $\mathrm{DBBC}$, we experiment 20 times and calculate the average of the 20 experimental results. Under each scheme (i.e., the ComBC, the CBBC, and the DBBC), WISP 

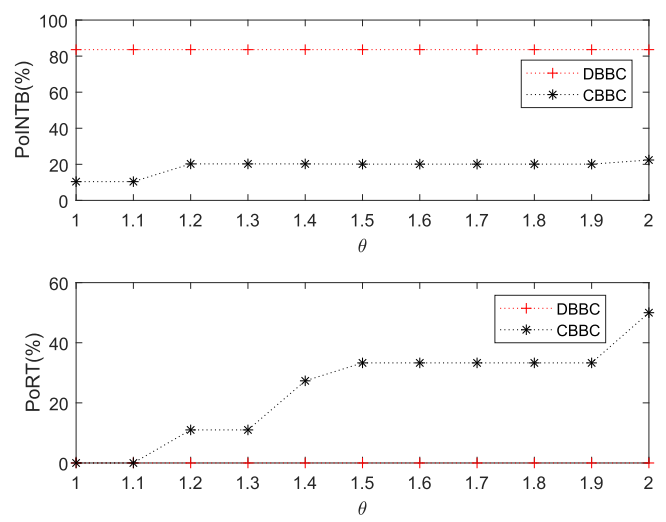

Fig. 6. PoINTB and PoRT vs $\theta$ when $\hat{m}=12$.
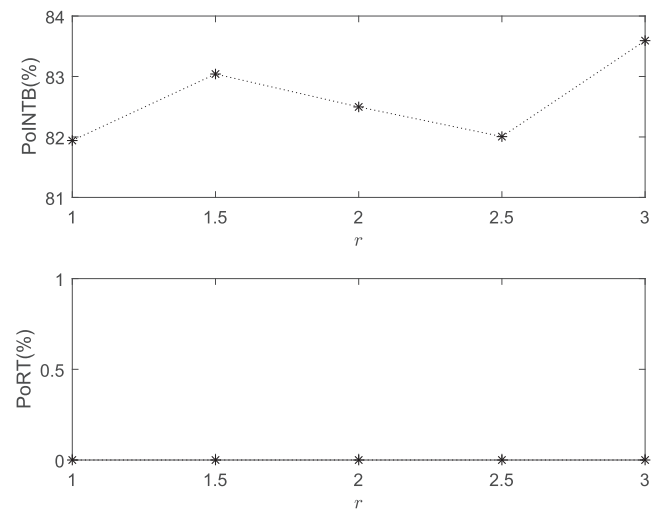

Fig. 7. PoINTB and PoRT vs $r$ when $\hat{m}=12$ and $\theta=1.6$.

A backscatters the same data in one peak-to-nadir period. The experimental results are shown in Figure 6.

From the figure, we have the following observations: First, the DBBC has PoINTB 83\%, exceeding nearly $60 \%$ compared with the CBBC. Second, the DBBC has PoRT close to 0, i.e., its throughput is close to that of the ComBC. Third, growth in $\theta$, i.e., relaxing the constraint on bit duration, the PoINTB and the PoRT of the DBBC are hardly impacted. In other words, the DBBC backscatters much more bits than the ComBC with the same energy, and it has almost the same throughput as the ComBC. The reason is that, in the DBBC, the group with more data blocks is transmitted with the maximum data rate, and the DBBC can apply the maximum data rate even when $\theta$ is small, thanks to the solution of the optimization problem for the DBBC. By the way, the CBBC has the PoINTB around $20 \%$, and the growth in $\theta$ causes the PoRT to increase, i.e., throughput decreases. The reason is that relaxing in bit duration encourages the $\mathrm{CBBC}$ to choose the lower data rate to backscatter data, which reduces throughput.

Next, we consider impact of data rates on PoINTB and PoRT by using data rate pair of $(r, r / 3)$, where we set $r=1.0,1.5,2.0,2.5,3.0 \mathrm{kbps}$. The experimental results are shown in Figure 7. From the figure, we observe that the variation in data rate hardly affects PoRT (see the lower part of the figure), i.e., throughput nearly keeps unchanged, and it only exerts slight impact on PoINTB (see the upper part of the figure). 
In summary, the $\mathrm{DBBC}$ can backscatter much more data than the ComBC and the $\mathrm{CBBC}$ with the same energy, and meanwhile the $\mathrm{DBBC}$ has the throughput close to the ComBC. That is, the proposed $\mathrm{DBBC}$ does not sacrifice throughput but the $\mathrm{CBBC}$ does.

\section{PERFORMANCE EVALUATION}

In this section, we evaluate energy-saving effectiveness of the proposed DBBC and study the impact of parameters on its performance. Meanwhile, we compare the DBBC with the CBBC [29], the PCBS [32], and the EEDDS [31] in terms of PoES and PoRT.

Considering there are no appropriate off-the-shelf nodes that can be tailored for experimenting different parameters, we apply simulation.

In the previous section, we obtain ECD $\alpha=1.64$ from the experiments on WISP that adopts FM0 (bi-phase space) baseband encoding. FM0 is widely applied in the backscattering communications [31]. Surely, ECD $\alpha$ may take different values in some other baseband transmission or carrier-modulation based transmission schemes. This encourages us to consider $\alpha=1.5,2.0,2.5$ in the simulation. In addition, the probabilities of bit 1 and bit 0 occurring in the original data may be different. For example, in the applications that adopt American Standard Code for Information Interchange (ASCII), the occurrence probability of bit 0 is appropriately equal to 0.56 [31]. Therefore, we consider $\beta=0.5,0.55,0.6$.

The simulation procedure contains the steps as follows, in which two data rates $r_{1}<r_{2}$ are applied in the sender to inform the receiver of which codebook is used for recovering the original data block.

Step 1. For given $\hat{m}, \alpha, \theta, r_{1}$, and $r_{2}$, solve the optimization problem in Equation (12) to find the solution that includes the optimal parameters of $m^{*}$ and $x^{*}$ and the optimal prefix codebooks $\mathbb{C}_{1}^{*}$ and $\mathbb{C}_{2}^{*}$. We assume $\left|\mathbb{C}_{1}^{*}\right|<\left|\mathbb{C}_{2}^{*}\right|$ without loss of generality (we can exchange $\mathbb{C}_{1}^{*}$ and $\mathbb{C}_{2}^{*}$ in the case when this inequality does not hold).

Step 2. Randomly generate the original data composed of $10^{6} \mathrm{~m}^{*}$ bits in which the proportion of bit 0 is $\beta$. (This can be realized by generating bit 0 with probability of $\beta$.)

Step 3. The sender divides the original data into $m^{*}$-bit blocks. For each block, the sender backscatters its corresponding codeword in $\mathbb{C}_{1}^{*} \cup \mathbb{C}_{2}^{*}$ to the receiver by using data rate $r_{i}$ if the backscattered codeword is in $\mathbb{C}_{i}^{*}, i=1,2$.

Step 4. Upon receiving a bit, the receiver tries decoding the original $m^{*}$-bit block by using codebook $\mathbb{C}_{i}^{*}$ if the sender applies data rate $r_{i}, i=1,2$.

Step 5. Accumulate the energy consumption resulting from delivering the codewords.

Step 6. Calculate the PoES and the PoRT after delivering all data.

Step 7. End.

It should be pointed out that, in the following simulations, two data rates of 3 and $1 \mathrm{kbps}$ are applied in the DBBC and the CBBC [29]. The ComBC backscatters data with the maximum data rate $3 \mathrm{kbps}$ for the sake of fair comparison.

To pay full attention to the impact of parameters of $\alpha$ and $\beta$ on DBBC, we first relax Constraint (12)(f) of optimization problem (12) (i.e., setting $\theta$ to a relatively large number). Then, setting $\beta=$ $0.5,0.55,0.6$, fixing $\hat{m}=12$, and letting $\alpha$ vary, we have Figure 8 . The figure indicates that: (1) the DBBC has the best PoES compared with the other schemes; (2) with a given $\beta$, PoES increases as $\alpha$ grows. In other words, a greater ECD brings more energy saving in the four schemes. This is because these schemes adopt $\operatorname{codebook}(\mathrm{s})$ and thus they can backscatter more LEBs instead of HEBs. In addition, setting $\alpha=1.5,2.0,2.5$, fixing $\hat{m}=12$, and letting $\beta$ vary yields Figure 9. From the figure, the DBBC still achieves the best PoES than the other three schemes and a greater $\beta$ brings in a higher PoES. The reason is that parameter $\beta$ represents the proportion of HEBs in the 


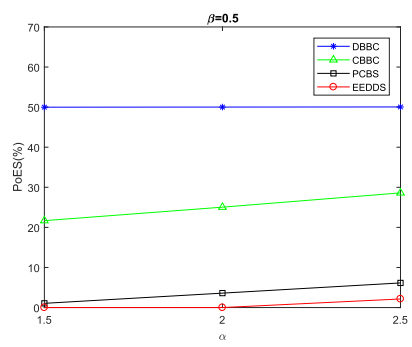

(a) PoES vs $\alpha$ when $\beta=0.5$

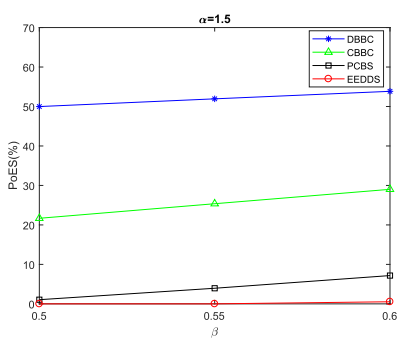

(a) PoES vs $\beta$ when $\alpha=1.5$

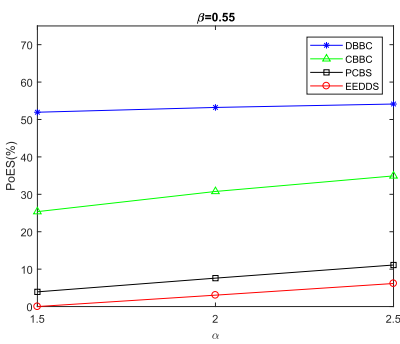

(b) PoES vs $\alpha$ when $\beta=0.55$

Fig. 8. Impact of $\alpha$ on PoES.

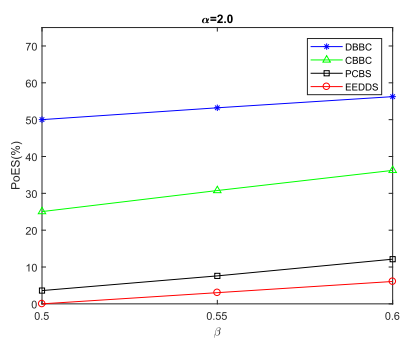

(b) PoES vs $\beta$ when $\alpha=2$

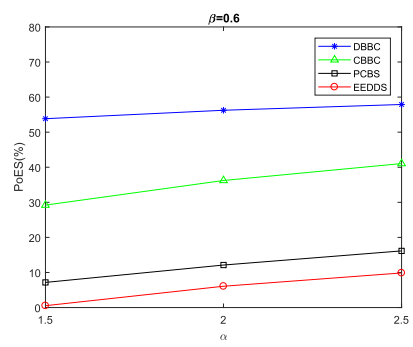

(c) PoES vs $\alpha$ when $\beta=0.6$

Fig. 9. Impact of $\beta$ on PoES.

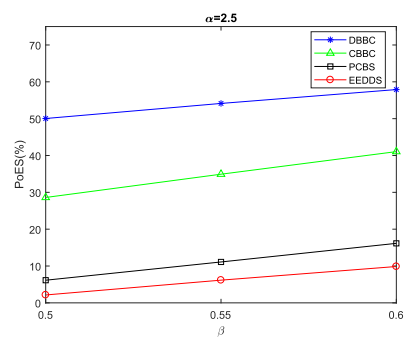

(c) PoES vs $\beta$ when $\alpha=2.5$

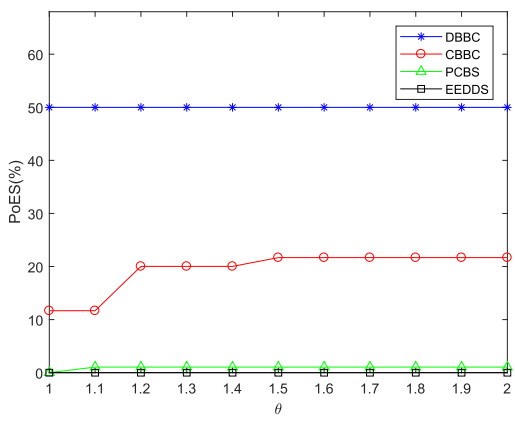

(a) PoES vs $\theta$

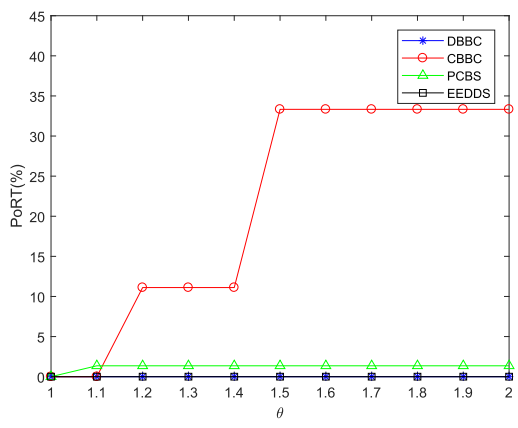

(b) PoRT vs $\theta$

Fig. 10. PoES and PoRT vs $\theta$ when $\alpha=1.5, \beta=0.5$ and $\hat{m}=12$.

original data, and a greater $\beta$ means that more HEBs contained in the original data so the four schemes, in which ECD-based codebook/codebooks is/are applied, can gain more energy saving.

Next, we let Constraint (12)(f) of optimization problem (12) function, i.e., we consider the impact of parameter $\theta$. With the view that bit duration is affected by variation of $\theta$, which influences throughput, we study PoRT in addition to PoES. Fixing $\alpha=1.5, \beta=0.5, \hat{m}=12$, and letting $\theta$ vary, we obtain Figure 10(a) and Figure 10(b), which show influence of $\theta$ on PoES and PoRT, respectively. From Figure 10(a), we observe that: (1) the DBBC achieves the maximum PoES; (2) growth of $\theta$ almost does not affect the PoES in the DBBC whereas a greater $\theta$ makes the CBBC save more energy. From Figure 10(b), we observe that the PoRT in the DBBC stays close to 0 , whereas that in the $\mathrm{CBBC}$ increases as $\theta$ grows. In other words, compared with the ComBC chosen as a baseline, the $\mathrm{DBBC}$ saves more energy than the $\mathrm{CBBC}$, and the $\mathrm{DBBC}$ almost does not sacrifice throughput 
but the $\mathrm{CBBC}$ does. The reason is that the $\mathrm{CBBC}$ applies a greater bit duration to save more energy when $\theta$ grows, thus reducing throughput.

\section{CONCLUSION}

The proposed DBBC makes use of the ECD existing in the traditional backscatter communications to design two prefix codebooks. By finding the two energy-efficient codebooks via the formulated energy consumption minimization problem, the DBBC can transmit much more data than the traditional backscatter communications with the same energy without sacrificing throughput. The DBBC can be implemented in the nodes with restricted memory. It is extremely suitable for the popular RFID systems. In practice, bit 0 and bit 1 occurring in the original bit stream may have different probabilities as in the case when ASCII is applied. For this case, an original $m$-bit block with a higher occurrence probability should be mapped into the codeword with a less energy consumption so more energy can be saved. Additionally, the bit error ratio of a backscatter communication link affects performance of the proposed scheme. We will investigate these problems in the future.

\section{REFERENCES}

[1] Dinesh Bharadia, Kiran R. Joshi, Manikanta Kotaru, and Sachin Katti. 2015. BackFi: High throughput Wi-Fi backscatter. In Proceedings of the ACM Special Interest Group on Data Communications Conference (SIGCOMM'15). 283-296.

[2] Kaikai Chi, Yi-Hua Zhu, Xiaohong Jiang, and Victor C. Leung. 2014. Energy-efficient prefix-free codes for wireless nano-sensor networks using ook modulation. IEEE Trans. Wirel. Commun. 13, 5 (May 2014), 2670-2682.

[3] Luis A. Fletscher, Luis A. Suárez, David Grace, Catalina V. Peroni, and José M. Maestre. 2019. Energy-aware resource management in heterogeneous cellular networks with hybrid energy sources. IEEE Trans. Netw. Serv. Manag. 16, 1 (Mar. 2019), 279-293.

[4] Wei Gong, Si Chen, and Jiangchuan Liu. 2017. Towards higher throughput rate adaptation for backscatter networks. In Proceedings of the IEEE International Conference on Network Protocols (ICNP'17). 1-10.

[5] Jinyu Hu, Juan Luo, Yanliu Zheng, and Keqin Li. 2019. Graphene-grid deployment in energy harvesting cooperative wireless sensor networks for green IoT. IEEE Trans. Ind. Inf. 15, 3 (Mar. 2019), 1820-1829.

[6] Pan Hu, Pengyu Zhang, and Deepak Ganesan. 2015. Laissez-Faire: Fully asymmetric backscatter communication. In Proceedings of the ACM Special Interest Group on Data Communications Conference (SIGCOMM'15). 255-267.

[7] EPCglobal Inc. 2004. EPC radio-frequency identity protocols Class-1 Generation-2 UHF RFID protocol for communications at 860-960 MHz. Retrieved from http://www.gs1.org/epcrfid/epc-rfid-uhf-air-interface-protocol/2-0-1.

[8] Vikram Iyer, Vamsi Talla, Bryce Kellogg, Shyamnath Gollakota, and Joshua R. Smith. 2016. Inter-technology backscatter: Towards Internet connectivity for implanted devices. In Proceedings of the ACM Special Interest Group on Data Communications Conference (SIGCOMM'16). 356-369.

[9] Meng Jin, Yuan He, Xin Meng, Dingyi Fang, and Xiaojiang Chen. 2018. Parallel backscatter in the wild: When burstiness and randomness play with you. In Proceedings of the 24th Annual International Conference on Mobile Computing and Networking (MobiCom'18). 471-485.

[10] Meng Jin, Yuan He, Xin Meng, Yilun Zheng, Dingyi Fang, and Xiaojiang Chen. 2017. FlipTracer: Practical parallel decoding for backscatter communication. In Proceedings of the 23rd Annual International Conference on Mobile Computing and Networking (MobiCom'17). 275-287.

[11] Bryce Kellogg, Aaron Parks, Shyamnath Gollakota, Joshua R. Smith, and David Wetherall. 2014. Wi-fi backscatter: Internet connectivity for RF-powered devices. In Proceedings of the ACM Special Interest Group on Data Communications Conference (SIGCOMM'14). 607-618.

[12] Yan Li, Zicheng Chi, Xin Liu, and Ting Zhu. 2018. Passive-ZigBee: Enabling Zigbee communication in IoT networks with $1000 \mathrm{x}+$ less power consumption. In Proceedings of the ACM Conference on Embedded Networked Sensor Systems (SenSys'18). 159-171.

[13] Vincent Liu, Aaron Parks, Vamsi Talla, Shyamnath Gollakota, David Wetherall, and Joshua R. Smith.2013. Ambient backscatter: Wireless communication out of thin air. In Proceedings of the ACM Special Interest Group on Data Communications Conference (SIGCOMM'13). 39-50.

[14] Bin Lyu, Changsheng You, Zhen Yang, and Guan Gui. 2018. The optimal control policy for RF-powered backscatter communication networks. IEEE Trans. Veh. Technol. 67, 3 (Mar. 2018), 2804-2808. 
[15] Saman Naderiparizi, Mehrdad Hessar, Vamsi Talla, Shyam Gollakota, and Joshua R. Smith. 2018. Towards batteryfree HD video streaming. In Proceedings of the USENIX Symposium on Networked Systems Design and Implementation (NSDI'18). 233-247.

[16] Saman Naderiparizi, Aaron N. Parks, Zerina Kapetanovic, Benjamin Ransford, and Joshua R. Smith. 2015. WISPCam: A battery-free RFID camera. In Proceedings of the IEEE Conference on Radio Frequency Identifcation (RFID'15). 166-173.

[17] Saman Naderiparizi, Yi Zhao, James Youngquist, Alanson P. Sample, and Joshua R. Smith. 2015. Self-localizing batteryfree cameras. In Proceedings of the ACM International foint Conference Pervasive Ubiquitous Computing (UbiComp'15). 445-449.

[18] Jiajue Ou, Mo Li, and Yuanqing Zheng. 2015. Come and be served: Parallel decoding for COTS RFID tags. In Proceedings of the 21st Annual International Conference on Mobile Computing and Networking (MobiCom'15). 500-511.

[19] Aaron N. Parks, Angli Liu, Shyamnath Gollakota, and Joshua R. Smith. 2014. Turbocharging ambient backscatter communication. In Proceedings of the ACM Special Interest Group on Data Communications Conference (SIGCOMM'14). 619-630.

[20] Jing Qian, Aaron N. Parks, Joshua R. Smith, Feifei Gao, and Shi Jin. 2019. IoT communications with M-PSK modulated ambient backscatter: Algorithm, analysis, and implementation. IEEE Internet Things 7. 6, 1 (Feb. 2019), 844-855.

[21] Alanson P. Sample, Daniel J. Yeager, Pauline S. Powledge, Alexander V. Mamishev, and Joshua R. Smith. 2008. Design of an RFID-based battery-free programmable sensing platform. IEEE Trans. Instrum. Meas. 57, 11 (Nov. 2008), 26082615.

[22] Kirubaveni Savarimuthu, Radha Sankararajan, and Sudha Murugesan. 2017. Analysis and design of power conditioning circuit for piezoelectric vibration energy harvester. IET Sci. Meas. Technol. 11, 6 (Mar. 2017), 723-730.

[23] Minchul Shin and Inwhee Joe. 2016. Energy management algorithm for solar-powered energy harvesting wireless sensor node for Internet of Things. IET Commun. 10, 12 (Mar. 2016), 1508-1521.

[24] Vamsi Talla, Mehrdad Hessar, Bryce Kellogg, Ali Najafi, Joshua R. Smith, and Shyam Gollakota. 2017. LoRa backscatter: Enabling the vision of ubiquitous connectivity. Proc. ACM Interact. Mob. Wear. Ubiq. Technol. 1, 3 (Sep. 2017), 105:1-105:24.

[25] Vamsi Talla, Bryce Kellogg, Shyamnath Gollakota, and Joshua R. Smith. 2017. Battery-free cellphone. Proc. ACM Interact. Mob. Wear. Ubiq. Technol. 1, 2 (June 2017), 25:1-25:20.

[26] Vamsi Talla and Joshua R. Smith. 2013. Hybrid analog-digital backscatter: A new approach for battery-free sensing. In Proceedings of the IEEE Conference on Radio Frequency Identifcation (RFID'13). 74-81.

[27] Cong Wang, Ji Li, Yuanyuan Yang, and Fan Ye. 2018. Combining solar energy harvesting with wireless charging for hybrid wireless sensor networks. IEEE Trans. Mobile Comput. 17, 3 (Mar. 2018), 560-576.

[28] Gang Yang, Ying-Chang Liang, Rui Zhang, and Yiyang Pei. 2018. Modulation in the air: Backscatter communication over ambient OFDM carrier. IEEE Trans. Commun. 66, 3 (Mar. 2018), 1219-1233.

[29] Yufan Zhang, Ertao Li, Yi-Hua Zhu, Kaikai Chi, and Xianzhong Tian. 2019. Energy-efficient prefix code based backscatter communications for wirelessly powered networks. IEEE Wireless Commun. Lett. 8, 2 (Apr. 2019), 348351.

[30] Yi Zhao, Joshua R. Smith, and Alanson Sample. 2015. NFC-WISP: A sensing and computationally enhanced near-field RFID platform. In Proceedings of the IEEE Conference on Radio Frequency Identifcation (RFID'15). 174-181.

[31] Yi-Hua Zhu, Ertao Li, and Kaikai Chi. 2018. Encoding scheme to reduce energy consumption of delivering data in radio frequency powered battery-free wireless sensor networks. IEEE Trans. Veh. Technol. 67, 4 (Apr. 2018), 3085-3097.

[32] Yi-Hua Zhu, Ertao Li, Kaikai Chi, and Xianzhong Tian. 2018. Designing prefix code to save energy for wirelessly powered wireless sensor networks. IET Commun. 12, 17 (May 2018), 2137-2144.

Received March 2020; revised July 2020; accepted September 2020 